Article

\title{
Synthetic Studies on Bioactive Natural Polyketides: Intramolecular Nitrile Oxide-Olefin Cycloaddition Approach for Construction of a Macrolactone Skeleton of Macrosphelide B
}

\author{
Seung-Mann Paek ${ }^{1}$ and Young-Ger Suh ${ }^{2, *}$
}

1 College of Pharmacy, the Institute of Health Sciences, Gyeongsang National University, Jinju daero, Jinju, Gyeongnam, 660-751, Korea; E-Mail: million@gnu.ac.kr (S-M.P.)

2 College of Pharmacy, Seoul National University, Gwanak-ro 599, Gwanak-gu, Seoul 151-742, Korea

* Author to whom correspondence should be addressed; E-Mail: ygsuh@snu.ac.kr; Tel.: +82-2-880-7875; Fax: +82-2-888-0649.

Received: 27 April 2011; in revised form: 26 May 2011 / Accepted: 7 June 2011 /

Published: 10 June 2011

\begin{abstract}
Studies on the synthesis of macrosphelide B via an intramolecular nitrile oxideolefin cycloaddition (INOC) is described. In particular, an asymmetric INOC approach using phase transfer catalysts seems to be a potentially efficient and versatile procedure for the construction of the macrolactone skeleton of macrosphelide $\mathrm{B}$ in terms of facial selectivity. Our preliminary and unprecedented stereoselective procedure is anticipated to be usefully applied through further studies for the synthesis of the macrosphelide family.
\end{abstract}

Keywords: intramolecular nitrile oxide cycloaddition; macrosphelide; polyketide; total synthesis; phase transfer catalyst

\section{Introduction}

Macrocyclic polyketides are considered promising natural resources for development of biologically active molecules, including new medicines [1], although many of them consist of a synthetically labile framework. In particular, the macrocyclic ring-closure reaction has been frequently a formidable problem, due to the unfavorable enthalpy or entropy [2]. Thus, a number of synthetic 
methods such as a mixed anhydride mediated macrolactonization [3], $\operatorname{Pd}(0)$-catalyzed cross coupling [4] and ring-closing metathesis [5,6] have been developed to overcome the intrinsic cyclization problem. Recently, the so-called intramolecular nitrile oxide-olefin cycloaddition (INOC, Scheme 1) approach has turned out as an alternative procedure for the requisite cyclization. $[7,8]$

INOC $[9,10]$ can serve as an excellent pathway for the generation of the new chiralities along with the ring-closure operation [11], because it proceeds through a concerted mechanism. Indeed, a large number of INOC approaches have been reported as good procedures for the synthesis of medium-sized cyclic skeletons from a viewpoint of efficient chiral transfer and ring-closure [12]. However, few macrocyclic ring-closure reactions employing INOC have been reported.

Scheme 1. Nitrile oxide cycloaddition.

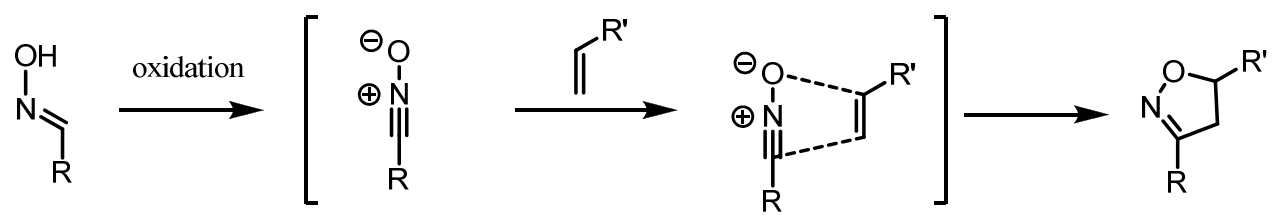

Recently, we reported an asymmetric total synthesis of macrosphelides (MSP) [13-15], which have been attracting interest from both biologists and chemists because of their potent anticancer, apoptotic, and immunosuppressant activities as well as unique structural features [16-21]. In contrast to the synthesis of macrosphelide A (MSPA) via Yamaguchi macrolactonization [3], synthesis of macrosphelide B (MSPB) employed INOC for construction of the macrocyclic skeleton, which avoided the undesired side reactions, such as epimerization or formation of the geometric isomer [13]. With this early success, we extended our work to a mechanistic study of INOC [14] and an investigation on its regio- and stereochemical control using chiral catalysts. In addition, we anticipated expanding its synthetic utilities to the structural variation of MSPs for further biological improvement [22-24]. The asymmetric INOC could be effectively applied to the asymmetric synthesis of MSPJ and MSPK [15]. Moreover, it would provide the regioselective ring formation for construction of the uncommon 15-membered macrolide framework of MSPM (Scheme 2). In this article, we describe our recent effort for new variants of the regio- and stereoselective INOC, utilized for MSPB synthesis.

\section{Results and Discussion}

Investigation on stereochemical control of INOC employed for the synthesis of MSPB (1) commenced with examination of various metal catalysts and the corresponding ligands. Aldoxime 4 is a precursor of the key intermediates 5 and 6 (Scheme 3) which can be efficiently transformed into MSPB (1) via a synthetic route developed by us [13]. It was also envisioned that successful stereocontrol of INOC would provide an invaluable transformation for MSP syntheses. 
Scheme 2. Structures of macrosphelides and INOC application for their syntheses.<smiles>C/C=C\C(=O)O[C@H](C)C(C)OC(=O)/C=C/C(O)C(C)OC(=O)/C=C\[C@H](C)O</smiles>

Macrosphelide A<smiles>C/C=C/[C@H](O)C(C)OC(=O)/C=C\C(=O)OC(C)CC(=O)OC(C)C(C)O</smiles>

Macrosphelide E<smiles>CC(C)OC(=O)CCC(O)C(C)OC(=O)CC(C)OC(=O)/C=C\[C@H](O)C(C)O</smiles>

Macrosphelide I<smiles>C/C=C/C(=O)C(C)OC(=O)C[C@H](C)OC(=O)/C=C/C(=O)OC(C)[C@H](C)O</smiles>

Macrosphelide B<smiles>C/C=C\CC(C)OC(=O)/C=C\C(=O)OC(C)CC(=O)OC(C)[C@H](O)/C=C\C</smiles>

Macrosphelide F

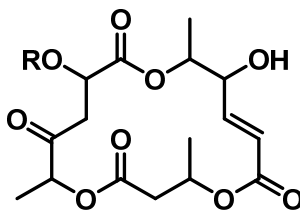

$\mathrm{R}=$ Me Macrosphelide $\mathrm{J}$ $\mathrm{R}=\mathrm{Et}$ Macrosphelide $\mathrm{K}$<smiles>C/C=C/C=C/[C@H](O)C(C)OC(=O)C[C@@H](C)COC(=O)/C=C\CC(C)OC</smiles>

Macrosphelide C<smiles>CC(/C=C\C(=O)O)CC/C=C/C(=O)OC(C)CC(=O)OC(C)C</smiles>

Macrosphelide G<smiles>C=C(/C=C\[C@H](O)C(C)OC(=O)CC(=O)CC(C)OC(=O)CC(C)OC(=O)CC)OC</smiles>

Macrosphelide L<smiles>CC(O)/C=C\C(=O)OC(C)CC(=O)OC(C)C(O)/C=C/C(=O)OC(C)C(C)O</smiles>

Macrosphelide D<smiles>CC(=O)CCOC(=O)C=C[C@@H](O)C(C)OC(=O)C=CCC(C)C</smiles>

Macrosphelide H<smiles>C/C=C\[C@H](O)C(C)OC(=O)/C=C\C(=O)OC(C)CC(=O)OC(C)C(C)O</smiles>

Macrosphelide M

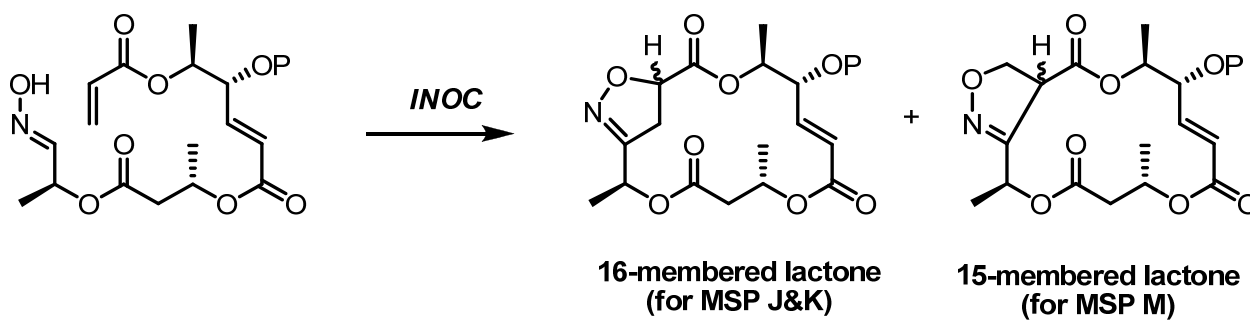

Scheme 3. Synthetic route to MSPB via INOC.

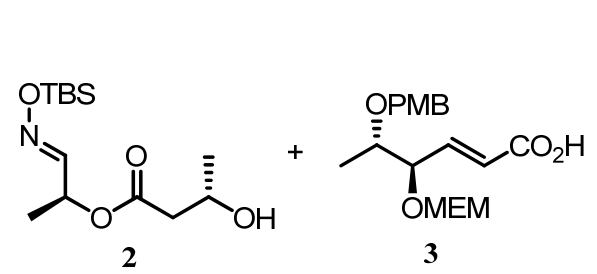

1. 2,4,6-trichlorobenzoylchloride,

$\mathrm{Et}_{3} \mathrm{~N}$, DMAP, tolene, $120^{\circ} \mathrm{C}, 3 \mathrm{~h}$

2. DDQ, $\mathrm{CH}_{2} \mathrm{Cl}_{2} / \mathrm{pH} 7$ buffer (10:1), rt, $2 \mathrm{~h}$

3. acryloyl chloride, $i \mathrm{Pr}_{2} \mathrm{NEt}, \mathrm{CH}_{2} \mathrm{Cl}_{2}, 0{ }^{\circ} \mathrm{C}$ to rt, $5 \mathrm{~h}$

4. TBAF, AcOH, rt, 10min (reference 13)

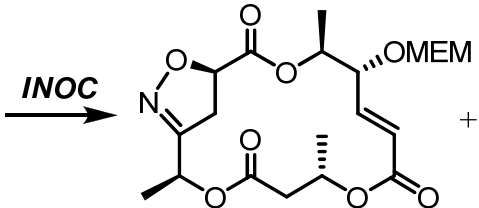

5<smiles>CO[C@H](C)[C@@H](/C=C/C(=O)O[C@@H](C)CC(=O)OC(C)C1=NO[C@H](C(=O)OC(C)C)C1)OC</smiles>

6
1. $\mathrm{Mo}(\mathrm{CO})_{6}, \mathrm{CH}_{3} \mathrm{CN} / \mathrm{H}_{2} \mathrm{O}, 80^{\circ} \mathrm{C}, 12 \mathrm{~h}$

2. Burgess reagent, benzene, $80^{\circ} \mathrm{C}, 14 \mathrm{~h}$

3. TFA, $\underset{\mathrm{CH}_{2} \mathrm{Cl}_{2}, \mathrm{rt}, 12 \mathrm{~h}}{\longrightarrow}$

(reference 13)<smiles>C=CC(=O)O[C@H](C)[C@H](/C=C/C(=O)O[C@H](C)CC(=O)O[C@@H](C)/C=N/O)OC</smiles>

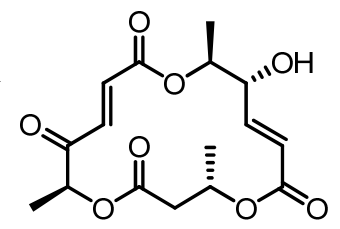

MSPB 1

Our initial study on stereocontrol of INOC by metal catalysts for the synthesis of MSPB is summarized in Scheme 4. As reported earlier, [13] a simple addition of aqueous $\mathrm{NaOCl}$ to oxime precursor 4 afforded a mixture of 5 and $\mathbf{6}$ in a good yield and with moderate facial selectivity. [25] Considering the dramatic increase of diastereo- and enantioselectivity by various metal cations with chiral ligands in nitrile oxide cycloaddition [11], we have also examined metal cations (entries 2-4). 
However, no significant improvement of diastereomeric ratio with decrease of chemical yields was observed. Addition of chiral ligands was not beneficial either, although $(R)$-BINOL induced a slight increase of facial selectivity. Interestingly, addition of Corey phase-transfer catalyst [26] under cycloaddition conditions afforded an inversion of facial selectivity to produce isoxazoline $\mathbf{6}$ as a major product although the moderate selectivity still hampered practical use of this condition. To the best of our knowledge, PTC-induced reverse facial selectivity in the INOC-mediated macro ring-closure reaction has not been reported yet.

Scheme 4. INOC of aldoxime 4 in the presence of various catalysts.

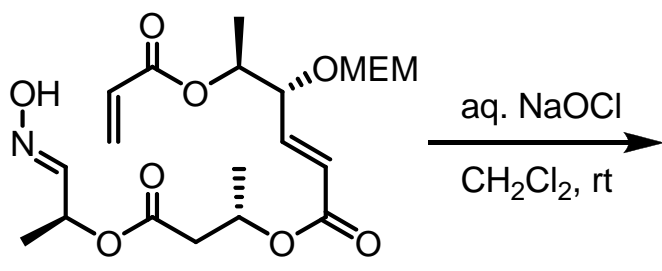

4<smiles>CO[C@H](/C=C/C(=O)O[C@H](C)CC(=O)OC(C)C1=NOC(C(=O)OC(C)C)C1)C(C)O</smiles>

5<smiles>COC(=O)C1=NO[C@@H](C(=O)O[C@H](C)CC(=O)O[C@H](C)/C=C/C(=O)O[C@@H](C)C(C)O)C1</smiles>

6

\begin{tabular}{ccccc}
\hline entry & catalyst & ligand & ${\text { yield }(5+6)^{\mathrm{a}}}^{\mathrm{a}}$ & ratio (5:6 $)^{\mathrm{a}}$ \\
\hline 1 & - & - & $96 \%$ & $2.0: 1$ \\
2 & $\mathrm{Ti}(i \mathrm{PrO})_{4}$ & - & $60 \%$ & $1.5: 1$ \\
3 & $\mathrm{MgBr}_{2}-\mathrm{OEt}_{2}$ & - & $64 \%$ & $1.7: 1$ \\
4 & $\mathrm{ZnBr}_{2}$ & - & $52 \%$ & $1.6: 1$ \\
5 & $\mathrm{ZnBr}_{2}$ & $(R)-\mathrm{BINOL}$ & $56 \%$ & $2.5: 1$ \\
6 & $\mathrm{ZnBr}_{2}$ & $(S)-\mathrm{BINOL}$ & $64 \%$ & $2.2: 1$ \\
7 & Corey catalyst & - & $72 \%$ & $1: 1.2$ \\
\hline
\end{tabular}

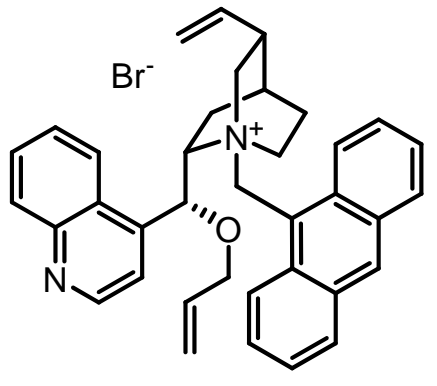

Corey catalyst

a. isolated results

Inspired with the interesting result from the PTC-assisted INOC, we intensively examined other PTC catalysts [27] for improved selectivity, as summarized in Scheme 5. Although other PTCs seemed not to influence much the facial selectivity of INOC, potential chiral induction by PTC in INOC was observed. In most cases, high chemical yields were observed with a variety of stereoselectivities. The reason for the change of facial selectivity is not clear at present. However, it is likely due to an effect of the coexistence of water and $\mathrm{CH}_{2} \mathrm{Cl}_{2}$ as solvents. 
Scheme 5. INOC of aldoxime 4 in the presence of various PTCs.

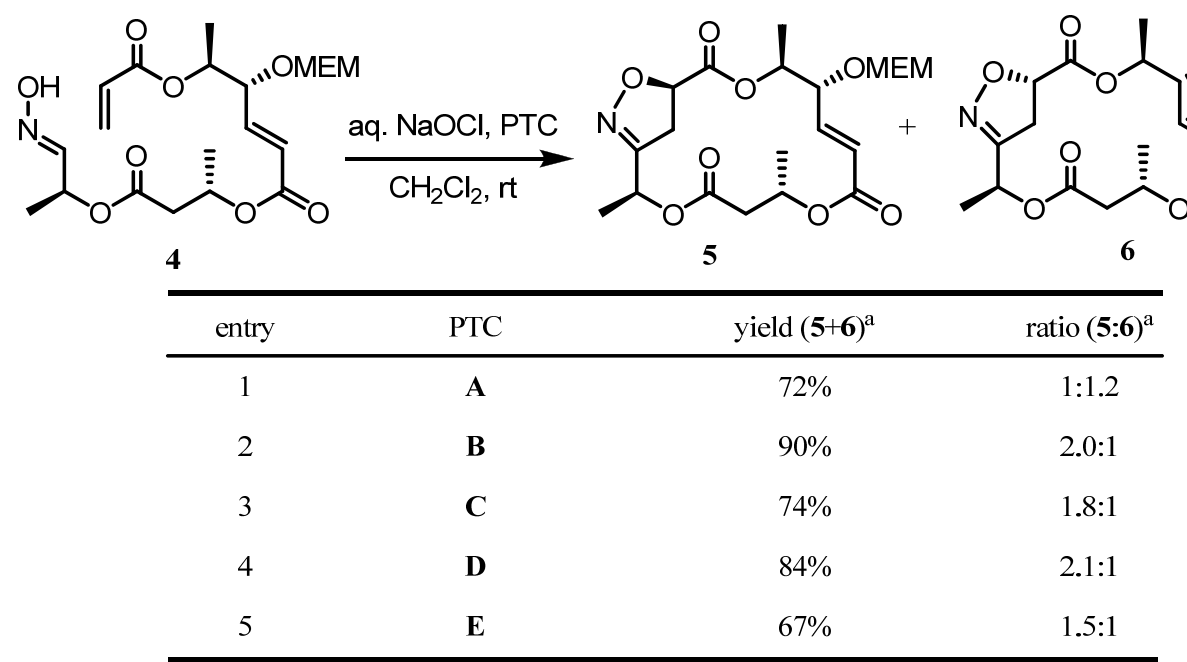

a. isolated yields

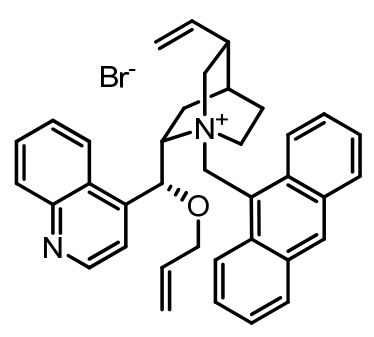

A

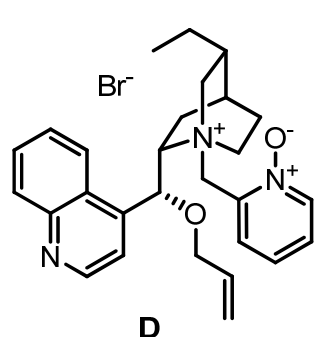

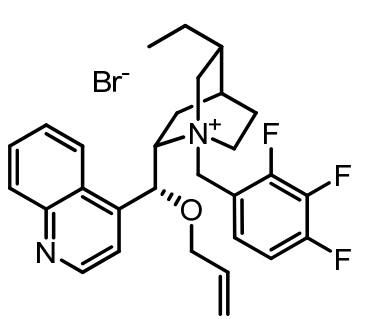

B<smiles>C=CCO[C@H](CN1CCCC1CC)c1ccnc2ccccc12</smiles>

C

Ioxazolines 5 and 6 could be efficiently transformed to MSPB, MSPJ and MSPK through appropriate synthetic sequences as shown in Scheme $6[13,28]$.

Scheme 6. Conversion of isoxazoline intermediates to macrosphelides.

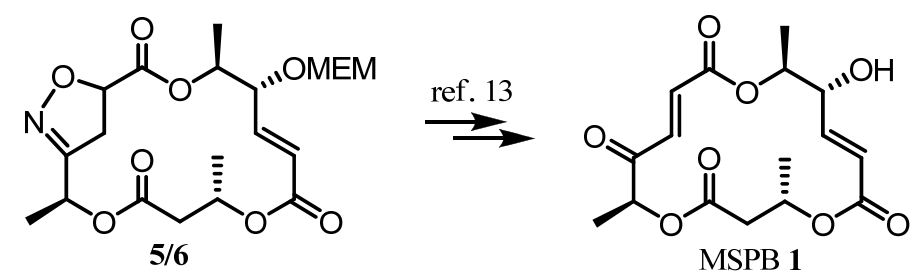

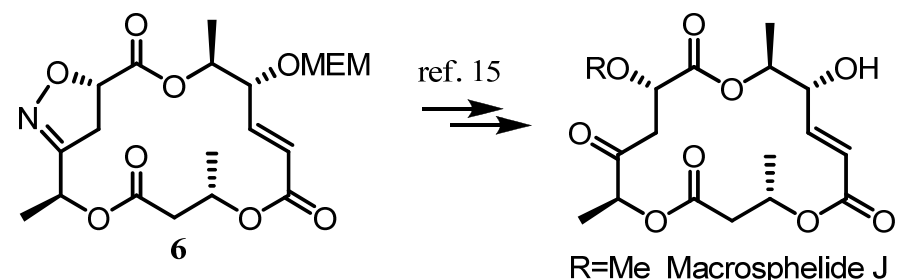




\section{Experimental}

\subsection{General}

Unless noted otherwise, all starting materials and reagents were obtained from commercial suppliers and were used without further purification. Tetrahydrofuran and $\mathrm{Et}_{2} \mathrm{O}$ were distilled from sodium benzophenone ketyl. Dichloromethane, triethylamine, acetonitrile and pyridine were freshly distilled from calcium hydride. All solvents used for routine isolation of products and chromatography were reagent grade and glass distilled. Reaction flasks were dried at $100{ }^{\circ} \mathrm{C}$. Air and moisture sensitive reactions were performed under argon atmosphere. Flash column chromatography was performed using silica gel 60 (230-400 mesh) with the indicated solvents. Thin-layer chromatography was performed using $0.25 \mathrm{~mm}$ silica gel plates. Optical rotations were measured on JASCO DIP 1000 digital polarimeter using $100 \mathrm{~nm}$ cell of $1 \sim 2 \mathrm{~mL}$ capacity. Chemical shifts are expressed in parts per million (ppm, $\delta$ ) downfield from tetramethylsilane and are referenced to the deuterated solvent $\left(\mathrm{CHCl}_{3}\right) .{ }^{1} \mathrm{H}-\mathrm{NMR}$ data (on Bruker JEOL LNM-LA 300, JEOL JNM-GCX 400 and AMX-500 MHz systems) are reported in the order of chemical shift, multiplicity (s, singlet; d, doublet; $t$, triplet; $q$, quartet; m, multiplet and/or multiple resonance), number of protons, and coupling constant in $\operatorname{Hertz}(\mathrm{Hz})$.

(1S)-2-([tert-Butyl(dimethyl)silyl]oxyimino)-1-methylethyl (3S)-3-[(4-methoxybenzyl)oxy] butanoate (2). To a solution of (S)-2-(4-methoxybenzyloxy)propanal O-tert-butyldimethylsilyl oxime [13] (2.0 g, $6.1 \mathrm{mmol})$ in $\mathrm{CH}_{2} \mathrm{Cl}_{2} /$ phosphate buffer solution $(10: 1,88 \mathrm{~mL}, \mathrm{pH} 7.0)$ at ambient temperature was added DDQ (2.1 g, $9.2 \mathrm{mmol}$ ). The reaction mixture was stirred for $1 \mathrm{~h}$, diluted with $\mathrm{CH}_{2} \mathrm{Cl}_{2}$ and filtered under reduced pressure. The organic layer was washed with $\mathrm{H}_{2} \mathrm{O}$ and aqueous layer was extracted with $\mathrm{Et}_{2} \mathrm{O}$. The combined organic layers were dried over $\mathrm{MgSO}_{4}$ and concentrated in vacuo. The residue was purified by flash column chromatography on silica gel $\left(\mathrm{Et}_{2} \mathrm{O}-n\right.$-hexane $\left.=1: 3\right)$ to afford $1.8 \mathrm{~g}$ of alcohol $\mathbf{1 6}$ and $p$-anisaldehyde as the inseparable mixture. This crude mixture was thus used for next step without further purification. To a solution of crude mixture (1.8 g) and (S)-3-(4methoxybenzyloxy)butanoic acid [13] $(1.3 \mathrm{~g}, 6.1 \mathrm{mmol})$ in $\mathrm{CH}_{2} \mathrm{Cl}_{2}(50 \mathrm{~mL})$ at ambient temperature were added EDCI (1.1 g, $6.1 \mathrm{mmol})$ and DMAP $(750 \mathrm{mg}, 6.1 \mathrm{mmol})$. After stirring for $12 \mathrm{~h}$, the reaction mixture was quenched with $\mathrm{H}_{2} \mathrm{O}$, extracted with $\mathrm{CH}_{2} \mathrm{Cl}_{2}$ and aqueous layer was extracted with EtOAc. The combined organic layers were dried over $\mathrm{MgSO}_{4}$ and concentrated in vacuo. The residue was purified by flash column chromatography on silica gel (EtOAc- $n$-hexane $=1: 9$ ) to afford $1.7 \mathrm{~g}$ (70\%) of $(S)$-[(S)-1-(tert-butyldimethylsilyloxyimino)propan-2-yl)] 3-hydroxybutanoate as a colorless oil: ${ }^{1} \mathrm{H}-\mathrm{NMR}\left(\mathrm{CDCl}_{3}, 300 \mathrm{MHz}, E / Z\right.$ mixture of aldoxime ethers): $\delta 7.33,6.67(\mathrm{~d}, 1 \mathrm{H}, J=5.1 \mathrm{~Hz})$, $7.08(\mathrm{~d}, 2 \mathrm{H}, J=8.6 \mathrm{~Hz}), 6.67(\mathrm{~d}, 2 \mathrm{H}, J=8.8 \mathrm{~Hz}), 5.74,5.32(\mathrm{dq}, 1 \mathrm{H}, J=5.1,6.6 \mathrm{~Hz}), 4.30(\mathrm{q}, 2 \mathrm{H}, J=$ $10.9 \mathrm{~Hz}), 3.83(\mathrm{~m}, 1 \mathrm{H}), 3.63(\mathrm{~s}, 3 \mathrm{H}), 2.52-2.23(\mathrm{~m}, 2 \mathrm{H}), 1.23(\mathrm{~d}, 3 \mathrm{H}, J=6.3 \mathrm{~Hz}), 1.08(\mathrm{~d}, 3 \mathrm{H}, J=6.0$ $\mathrm{Hz}), 0.76(\mathrm{~s}, 9 \mathrm{H}), 0.00(\mathrm{~m}, 6 \mathrm{H}) ;{ }^{13} \mathrm{C}-\mathrm{NMR}\left(\mathrm{CDCl}_{3}, 100 \mathrm{MHz}, E / Z\right.$ mixture of aldoxime ethers) $\delta 170.4$, 159.1, 155.4, 153.3, 129.2, 113.7, 77.2, 71.6, 71.5, 70.6, 70.5, 67.9, 65.8, 55.2, 42.0 , 25.9 , 19.8, 18.1, 17.8, 16.9, -5.3, -5.4; IR (neat) $v_{\max } 2932,2857,1741,1613,1513,1464,1373,1298 \mathrm{~cm}^{-1}$; LRMS (FAB) $m / z 409\left(\mathrm{M}^{+}\right)$. 
To a solution of the $(S)$-((S)-1-(tert-butyldimethylsilyloxyimino)propan-2-yl) 3-hydroxybutanoate (1.1 g, $2.7 \mathrm{mmol})$ in $\mathrm{CH}_{2} \mathrm{Cl}_{2} /$ phosphate buffer solution $(20: 1,32 \mathrm{~mL}, \mathrm{pH} 7.0)$ at ambient temperature was added DDQ $(670 \mathrm{mg}, 2.9 \mathrm{mmol})$. The reaction mixture was stirred for $2 \mathrm{~h}$ and the reaction mixture was diluted with $\mathrm{CH}_{2} \mathrm{Cl}_{2}$, filtered under reduced pressure. The organic layer was washed with $\mathrm{H}_{2} \mathrm{O}$, dried over $\mathrm{MgSO}_{4}$ and concentrated in vacuo. The residue was purified by flash column chromatography on silica gel (EtOAc- $n$-hexane $=1: 5)$ to afford $650 \mathrm{mg}(84 \%)$ of alcohol 2 as a colorless oil. ${ }^{1} \mathrm{H}-\mathrm{NMR}\left(\mathrm{CDCl}_{3}, 300 \mathrm{MHz}, E / Z\right.$ mixture of aldoxime ethers) $\delta 7.46,6.84(\mathrm{~d}, 1 \mathrm{H}, J=4.9$ $\mathrm{Hz}), 5.91,5.48(\mathrm{dq}, 1 \mathrm{H}, J=4.9,6.7 \mathrm{~Hz}), 4.17$ (bs, 1H), 2.96, 2.87 (d, 1H, $J=3.3 \mathrm{~Hz}), 2.51-2.36$ (m, $2 \mathrm{H}), 1.39(\mathrm{~d}, 3 \mathrm{H}, J=6.7 \mathrm{~Hz}), 1.20(\mathrm{~d}, 3 \mathrm{H}, J=6.2 \mathrm{~Hz}), 0.89(\mathrm{~s}, 9 \mathrm{H}), 0.14(\mathrm{~m}, 6 \mathrm{H}) ;{ }^{13} \mathrm{C}-\mathrm{NMR}\left(\mathrm{CDCl}_{3}\right.$, $100 \mathrm{MHz}, E / Z$ mixture of aldoxime ethers) $\delta 171.9,171.8,153.0,68.1,65.9,64.2,64.1,42.9,42.7$, 25.9, 22.4, 18.1, 18.0, 17.8, 17.0, -5.1, -5.3; IR (neat) $v_{\max } 3435,2933,2858,1738,1465,1375,1253$, $1175 \mathrm{~cm}^{-1}$; LRMS (FAB) $\mathrm{m} / z 290\left(\mathrm{M}+\mathrm{H}^{+}\right.$); HRMS (FAB) Calcd for $\mathrm{C}_{13} \mathrm{H}_{28} \mathrm{NO}_{4} \mathrm{Si}\left(\mathrm{M}+\mathrm{H}^{+}\right)$: 290.1788, Found 290.1791.

(E,4R,5S)-4-((2-methoxyethoxy)methoxy)-5-(4-methoxybenzyloxy)hex-2-enoic acid (3). To a solution of (3R,4S,E)-4-(4-methoxybenzyloxy)-1-(4-methyl-2,6,7-trioxabicyclo[2.2.2]octan-1-yl)pent-1-en-3-ol [13] $(87 \mathrm{mg}, 0.25 \mathrm{mmol})$ in $\mathrm{CH}_{2} \mathrm{Cl}_{2}(5 \mathrm{~mL})$ were added $i-\mathrm{Pr}_{2} \mathrm{Net}(130 \mu \mathrm{L}, 0.75 \mathrm{mmol})$ and $\mathrm{MEMCl}$ $(56 \mu \mathrm{L}, 0.50 \mathrm{mmol})$ subsequently at $0{ }^{\circ} \mathrm{C}$. After stirring of the mixture for $5 \mathrm{~h}$ at the ambient temperature, additional $i$ - $\operatorname{Pr}_{2} \mathrm{NEt}(260 \mu \mathrm{L}, 1.5 \mathrm{mmol})$ and $\mathrm{MEMCl}(112 \mu \mathrm{L}, 1.0 \mathrm{mmol})$ were added subsequently at $0{ }^{\circ} \mathrm{C}$. The reaction mixture was stirred at ambient temperature for $36 \mathrm{~h}$ and quenched with $\mathrm{H}_{2} \mathrm{O}$. This mixture was extracted with $\mathrm{CH}_{2} \mathrm{Cl}_{2}$ and aqueous layer was extracted with EtOAc. The combined organic layers were dried over $\mathrm{Na}_{2} \mathrm{SO}_{4}$ and concentrated in vacuo. This crude mixture was used for next step without further purification. To a solution of MEM ether (190 mg, crude product) in THF $(4 \mathrm{~mL})$ and $\mathrm{H}_{2} \mathrm{O}(4 \mathrm{~mL})$ was added $1 \mathrm{~N} \mathrm{HCl}(0.1 \mathrm{~mL})$. The reaction mixture was stirred for $10 \mathrm{~min}$ at ambient temperature, and lithium hydroxide monohydrate $(80 \mathrm{mg}, 1.9 \mathrm{mmol})$ was added. After stirring for an additional $2 \mathrm{~h}$, the reaction mixture was acidified with $1 \mathrm{~N} \mathrm{HCl}(\mathrm{pH}<3)$ and extracted with EtOAc. The combined organic layers were washed with brine, dried over $\mathrm{MgSO}_{4}$ and concentrated in vacuo. The residue was purified by flash column chromatography on silica gel $\left(\mathrm{CH}_{2} \mathrm{Cl}_{2}-\mathrm{MeOH}=15: 1\right)$ to afford $76 \mathrm{mg}(86 \%$, for 2 steps $)$ of acid 3 as a colorless oil: $[\alpha]_{\mathrm{D}}^{20}-39.3$ (c 0.5, MeOH); ${ }^{1} \mathrm{H}-\mathrm{NMR}\left(\mathrm{CDCl}_{3}, 300 \mathrm{MHz}\right) \delta 7.17(\mathrm{~d}, 2 \mathrm{H}, J=8.6 \mathrm{~Hz}), 6.93(\mathrm{dd}, 1 \mathrm{H}, J=5.9,15.7$ $\mathrm{Hz}), 6.79(\mathrm{~d}, 2 \mathrm{H}, J=8.6 \mathrm{~Hz}), 5.99(\mathrm{dd}, 1 \mathrm{H}, J=1.5,15.7 \mathrm{~Hz}), 4.69$ (dd, 2H, $J=7.0,19.1 \mathrm{~Hz}), 4.44$ (s, $2 \mathrm{H}), 4.31-4.27(\mathrm{~m}, 1 \mathrm{H}), 3.75-3.65(\mathrm{~m}, 1 \mathrm{H}), 3.72(\mathrm{~s}, 3 \mathrm{H}), 3.65-3.47(\mathrm{~m}, 1 \mathrm{H}), 3.45-3.42(\mathrm{~m}, 2 \mathrm{H})$, $3.32-3.30(\mathrm{~m}, 1 \mathrm{H}), 3.30(\mathrm{~s}, 3 \mathrm{H}), 1.12(\mathrm{~d}, 3 \mathrm{H}, J=6.4 \mathrm{~Hz}) ;{ }^{13} \mathrm{C}-\mathrm{NMR}\left(\mathrm{CDCl}_{3}, 75 \mathrm{MHz}\right) \delta 170.6,159.1$, $147.8,130.2,129.2,113.7,94.2,77.6,76.0,71.6,70.8,67.2,58.9,55.2,15.6$; IR (neat) $v_{\max } 2935$, 1717, 1613, 1513, 1459, 1248, 1175, 1099, $1035 \mathrm{~cm}^{-1}$; LRMS (FAB) $m / z 355\left(\mathrm{M}+\mathrm{H}^{+}\right)$; HRMS (FAB) Calcd for $\mathrm{C}_{18} \mathrm{H}_{27} \mathrm{O}_{7}\left(\mathrm{M}+\mathrm{H}^{+}\right)$: 355.1757, Found 355.1765.

(4R,5S,E)-((S)-4-((S,E)-1-(hydroxyimino)propan-2-yloxy)-4-oxobutan-2-yl) 5-(acryloyloxy)-4-((2methoxyethoxy)methoxy)hex-2-enoate (4). To a solution of $(2 E, 4 R, 5 S)-((7 S, 11 S)-2,2,3,3,7-$ pentamethyl9-oxo-4,8-dioxa-5-aza-3-siladodec-5-en-11-yl) 5-(acryloyloxy)-4-((2-methoxyethoxy)methoxy)hex-2enoate [13] (86 mg, $0.15 \mathrm{mmol})$ in THF $(5 \mathrm{~mL})$ at ambient temperature were added AcOH (2 drops) and TBAF $(1.0 \mathrm{M}$ in THF, $0.23 \mathrm{~mL}, 0.23 \mathrm{mmol})$. The reaction mixture was stirred for $10 \mathrm{~min}$ and 
quenched with $\mathrm{H}_{2} \mathrm{O}$. The mixture was extracted with EtOAc and the combined organic layers were dried over $\mathrm{MgSO}_{4}$ and concentrated in vacuo. The residue was purified by flash column chromatography on silica gel (EtOAc- $n$-hexane $=1: 1)$ to afford $69 \mathrm{mg}(99 \%)$ of acryloyl oxime 4 as a colorless oil: ${ }^{1} \mathrm{H}-\mathrm{NMR}\left(\mathrm{CDCl}_{3}, 300 \mathrm{MHz}, E / Z\right.$ mixture of aldoximes) $\delta 7.98(\mathrm{~s}, 1 \mathrm{H}), 7.33(\mathrm{~d}, 1 \mathrm{H}$, $J=5.6 \mathrm{~Hz}), 6.71(\mathrm{dd}, 1 \mathrm{H}, J=6.0,15.7 \mathrm{~Hz}), 6.36(\mathrm{dd}, 1 \mathrm{H}, J=1.2,17.2 \mathrm{~Hz}), 6.09-6.00(\mathrm{~m}, 1 \mathrm{H}), 5.97$ $(\mathrm{dd}, 1 \mathrm{H}, J=1.2,15.7 \mathrm{~Hz}), 5.79(\mathrm{dd}, 1 \mathrm{H}, J=1.4,10.4 \mathrm{~Hz}), 5.40-5.36(\mathrm{~m}, 1 \mathrm{H}), 5.27-5.23(\mathrm{~m}, 1 \mathrm{H})$, $5.08-5.04(\mathrm{~m}, 1 \mathrm{H}), 4.67(\mathrm{dd}, 2 \mathrm{H}, J=6.9,17.7 \mathrm{~Hz}), 4.37-4.35(\mathrm{~m}, 2 \mathrm{H}), 3.74-3.68(\mathrm{~m}, 1 \mathrm{H})$, $3.61-3.54(\mathrm{~m}, 1 \mathrm{H}), 3.47-3.44(\mathrm{~m}, 2 \mathrm{H}), 3.31(\mathrm{~s}, 3 \mathrm{H}), 2.67-2.46(\mathrm{~m}, 2 \mathrm{H}), 1.32(\mathrm{~d}, 3 \mathrm{H}, J=6.5 \mathrm{~Hz})$, $1.28(\mathrm{~d}, 3 \mathrm{H}, J=6.2 \mathrm{~Hz}), 1.18(\mathrm{~d}, 3 \mathrm{H}, J=6.6 \mathrm{~Hz}), 0.89(\mathrm{~s}, 9 \mathrm{H}), 0.12(\mathrm{~s}, 6 \mathrm{H}) ;{ }^{13} \mathrm{C}-\mathrm{NMR}\left(\mathrm{CDCl}_{3}, 75\right.$ $\mathrm{MHz}, E / Z$ mixture of aldoximes) $\delta 169.2,164.8,149.6,143.3,131.3,128.3,124.1,93.8,77.2,76.3$, 71.6, 67.9, 67.2, 59.0, 41.0, 19.9, 17.9, 14.5; IR (neat) $v_{\max } 3395,2933,1725,1454,1407,1272,1193$, $1055 \mathrm{~cm}^{-1}$; LRMS (FAB) $\mathrm{m} / z 446\left(\mathrm{M}+\mathrm{H}^{+}\right)$; HRMS (FAB) Calcd for $\mathrm{C}_{20} \mathrm{H}_{32} \mathrm{NO}_{10}\left(\mathrm{M}+\mathrm{H}^{+}\right)$: 446.2026, Found 446.2028.

\subsection{Representative INOC Procedure}

To a solution of oxime $(2.5 \mathrm{mg}, 5.61 \mu \mathrm{mol})$ and Corey catalyst $\mathbf{A}(4.0 \mathrm{mg}, 6.60 \mu \mathrm{mol})$ in $\mathrm{CH}_{2} \mathrm{Cl}_{2}$ $(1.0 \mathrm{~mL})$ was added aqueous $\mathrm{NaOCl}(0.05 \mathrm{~mL}$, c.a. $10 \%)$ at ambient temperature. The reaction mixture was stirred for $1 \mathrm{~h}$ at the same temperature and diluted with $\mathrm{H}_{2} \mathrm{O}$. The aqueous layer was extracted with $\mathrm{CH}_{2} \mathrm{Cl}_{2}$ and the combined organic layers were dried over $\mathrm{MgSO}_{4}$ and concentrated in vacuo. The residue was purified by flash column chromatography on silica gel (EtOAc: $n$-hexane $=2: 3$ to $2: 1$ ) to afford $0.8 \mathrm{mg}$ of isoxazoline 5 and $1.0 \mathrm{mg}$ of isoxazoline 6 as a pale yellow oil, respectively.

(2S,6S,11R,12S,15R)-11-[(2-methoxyethoxy)methoxy]-2,6,12-trimethyl-3, 7,13,16-tetraoxa-17-azabicyclo[13.2.1] octadeca-1(17),9-diene-4,8,14-trione (5). [ $\alpha]_{\mathrm{D}}^{20}-107.5\left(\mathrm{c} 0.8, \mathrm{CHCl}_{3}\right) ;{ }^{1} \mathrm{H}-\mathrm{NMR}\left(\mathrm{CDCl}_{3}\right.$, $400 \mathrm{MHz}) \delta 6.68(\mathrm{dd}, 1 \mathrm{H}, J=4.7,15.7 \mathrm{~Hz}), 6.00(\mathrm{~d}, 1 \mathrm{H}, J=15.7 \mathrm{~Hz}), 5.47(\mathrm{q}, 1 \mathrm{H}, J=6.5 \mathrm{~Hz})$, $5.33-5.30(\mathrm{~m}, 1 \mathrm{H}), 5.00(\mathrm{dd}, 1 \mathrm{H}, J=7.5,11.7 \mathrm{~Hz}), 4.89(\mathrm{dq}, 1 \mathrm{H}, J=4.0,6.4 \mathrm{~Hz}), 4.69(\mathrm{dd}, 2 \mathrm{H}, J=$ 7.0, 13.2 Hz), $4.21(\mathrm{t}, 1 \mathrm{H}, J=8.0 \mathrm{~Hz}), 3.78-3.73(\mathrm{~m}, 1 \mathrm{H}), 3.64-3.59(\mathrm{~m}, 1 \mathrm{H}), 3.55-3.51(\mathrm{~m}, 2 \mathrm{H})$, $3.37(\mathrm{~s}, 3 \mathrm{H}), 3.22(\mathrm{dd}, 1 \mathrm{H}, J=11.9,17.6 \mathrm{~Hz}), 3.07(\mathrm{dd}, 1 \mathrm{H}, J=7.5,17.6 \mathrm{~Hz}), 2.69-2.59(\mathrm{~m}, 2 \mathrm{H})$, $1.56(\mathrm{~d}, 3 \mathrm{H}, J=6.6 \mathrm{~Hz}), 1.42(\mathrm{~d}, 3 \mathrm{H}, J=6.2 \mathrm{~Hz}), 1.33(\mathrm{~d}, 3 \mathrm{H}, J=6.3 \mathrm{~Hz}) ;{ }^{13} \mathrm{C}-\mathrm{NMR}\left(\mathrm{CDCl}_{3}, 100\right.$ $\mathrm{MHz}) \delta 170.3,169.1,164.5,157.4,144.5,125.1,93.6,78.1,77.2,77.1,72.3,71.5,67.9,67.4,65.9$, 59.0, 40.6, 40.4, 19.7, 17.4; IR (neat) $v_{\max } 2924,1739,1455,1371,1272,1187,1051,852 \mathrm{~cm}^{-1}$; LRMS (EI) $m / z 443\left(\mathrm{M}+\mathrm{Na}^{+}\right)$; HRMS (FAB) Calcd for $\mathrm{C}_{20} \mathrm{H}_{30} \mathrm{NO}_{10}\left(\mathrm{M}+\mathrm{H}^{+}\right)$: 444.1870, Found 444.1881.

(2S, 6S, 11R, 12S, 15S)-11-[(2-methoxyethoxy)methoxy]-2,6,12-trimethyl-3, 7, 13, 16-tetraoxa-17azabicyclo[13.2.1] octadeca-1(17),9-diene-4,8,14-trione (6). [ $\alpha]_{\mathrm{D}}^{20}-55.1$ (c 1.16, $\mathrm{CHCl}_{3}$ ); ${ }^{1} \mathrm{H}-\mathrm{NMR}$ $\left(\mathrm{CDCl}_{3}, 300 \mathrm{MHz}\right) \delta 6.67(\mathrm{dd}, 1 \mathrm{H}, J=7.8,15.9 \mathrm{~Hz}), 5.99(\mathrm{~d}, 1 \mathrm{H}, J=15.9 \mathrm{~Hz}), 5.77(\mathrm{q}, 1 \mathrm{H}, J=6.9$ $\mathrm{Hz}), 5.45-5.39(\mathrm{~m}, 1 \mathrm{H}), 4.95(\mathrm{dd}, 1 \mathrm{H}, J=8.4,12.2 \mathrm{~Hz}), 4.94(\mathrm{dq}, 1 \mathrm{H}, J=6.2,9.3 \mathrm{~Hz}), 4.74(\mathrm{dd}, 2 \mathrm{H}$, $J=7.1,16.1 \mathrm{~Hz}), 4.05(\mathrm{t}, 1 \mathrm{H}, J=8.7 \mathrm{~Hz}), 3.78-3.72(\mathrm{~m}, 1 \mathrm{H}), 3.65-3.58(\mathrm{~m}, 1 \mathrm{H}), 3.53-3.50$ $(\mathrm{m}, 2 \mathrm{H}), 3.38-3.26(\mathrm{~m}, 1 \mathrm{H}), 3.36(\mathrm{~s}, 3 \mathrm{H}), 2.82(\mathrm{dd}, 1 \mathrm{H}, J=8.2,17.5 \mathrm{~Hz}), 2.66-2.53(\mathrm{~m}, 2 \mathrm{H}), 1.48$ $(\mathrm{d}, 3 \mathrm{H}, J=6.7 \mathrm{~Hz}), 1.36(\mathrm{~d}, 3 \mathrm{H}, J=6.2 \mathrm{~Hz}), 1.30(\mathrm{~d}, 3 \mathrm{H}, J=6.3 \mathrm{~Hz}) ;{ }^{13} \mathrm{C}-\mathrm{NMR}\left(\mathrm{CDCl}_{3}, 125 \mathrm{MHz}\right)$ 
$\delta 169.6,167.8,164.0,158.3,145.3,125.3,93.8,77.6,76.4,71.5,71.4,68.1,67.4,66.5,58.9,40.9$, 39.0, 19.9, 19.1, 17.6; IR (neat) $v_{\max } 2925,2854,1742,1455,1374,1252,1192,1104,1051 \mathrm{~cm}^{-1}$; LRMS (FAB) $m / z 444\left(\mathrm{M}+\mathrm{H}^{+}\right)$; HRMS (FAB) Calcd for $\mathrm{C}_{20} \mathrm{H}_{30} \mathrm{NO}_{10}\left(\mathrm{M}+\mathrm{H}^{+}\right)$: 444.1870, Found 444.1854.

Macrosphelide B (1): To a solution of MEM protected macrosphelide B [21] (1.6 mg, $3.7 \mu \mathrm{mol})$ in $\mathrm{CH}_{2} \mathrm{Cl}_{2}(0.3 \mathrm{~mL})$ was added trifluoroacetic acid $(0.3 \mathrm{~mL})$ at ambient temperature. After stirring for $3 \mathrm{~h}$, the reaction mixture was concentrated in vacuo and purified by flash column chromatography on silica gel (EtOAc- $n$-hexane $=1: 2 \rightarrow 1: 1)$ to afford $1.2 \mathrm{mg}$ (94\%) of macrosphelide B (2) as a colorless oil: $[\alpha]_{\mathrm{D}}^{24}+10.8(\mathrm{c} 0.065, \mathrm{MeOH}) ;{ }^{1} \mathrm{H}-\mathrm{NMR}\left(\mathrm{CDCl}_{3}, 300 \mathrm{MHz}\right) \delta 6.96(\mathrm{~d}, 1 \mathrm{H}, J=15.7 \mathrm{~Hz}), 6.85(\mathrm{dd}, 1 \mathrm{H}$, $J=3.4,15.5 \mathrm{~Hz}), 6.68(\mathrm{~d}, 1 \mathrm{H}, J=15.5 \mathrm{~Hz}), 6.02(\mathrm{dd}, 1 \mathrm{H}, J=2.0,15.5 \mathrm{~Hz}), 5.43-5.36(\mathrm{~m}, 1 \mathrm{H})$, $5.04-4.95(\mathrm{~m}, 2 \mathrm{H}), 4.25(\mathrm{bs}, 1 \mathrm{H}), 2.76(\mathrm{dd}, 1 \mathrm{H}, J=10.9,16.1 \mathrm{~Hz}), 2.55(\mathrm{dd}, 1 \mathrm{H}, J=2.3,16.1 \mathrm{~Hz})$, $1.43(\mathrm{~d}, 3 \mathrm{H}, J=6.6 \mathrm{~Hz}), 1.37$ (d, 3H, $J=7.1 \mathrm{~Hz}), 1.29$ (d, 3H, $J=6.2 \mathrm{~Hz}) ;{ }^{13} \mathrm{C}-\mathrm{NMR}\left(\mathrm{CDCl}_{3}, 75\right.$ MHz) $\delta 196.2,170.3,165.4,164.1,144.1,132.5,132.0,122.5,77.2,75.8$, 74.8, 40.5, 19.8, 17.9, 16.1; IR (neat) $v_{\max } 3464,2924,1725,1539,1455,1265,1182,1057 \mathrm{~cm}^{-1}$; LRMS (FAB) $\mathrm{m} / z 341$ $\left(\mathrm{M}+\mathrm{H}^{+}\right)$; HRMS (FAB) Calcd for $\mathrm{C}_{16} \mathrm{H}_{21} \mathrm{O}_{8}\left(\mathrm{M}+\mathrm{H}^{+}\right)$: 341.1236, Found 341.1229.

\section{Conclusions}

We have investigated the catalyst-assisted stereocontrol of INOC in connection with the synthesis of MSPB. Although most of the cycloaddition conditions didn't provide excellent selectivities, it was notable that PTC-assisted INOC exhibited reverse selectivity. This unprecedented discovery was applied for synthesis of the MSP family as well as other natural polyketides. Further studies on mechanism, increase of facial selectivity and synthetic applications are currently ongoing.

\section{Acknowledgements}

This work was supported by a National Research Foundation of Korea (NRF) grant funded by the Korean government (MEST) (No. 2009-0083533) and in part by the Research Institute of Pharmaceutical Science in Seoul National University. We specially thank S.-S. Jew and H.-G. Park of the College of Pharmacy, Seoul National University for generous gifts for PTC catalysts.

\section{References and Notes}

1. Dewick, P.M. Medicinal Natural Products, 2nd ed.; Wiley and Sons: Sussex, UK, 2001.

2. Kang, E.J.; Lee, E. Total synthesis of oxacyclic macrodiolide natural products. Chem. Rev. 2005, 105, 4343-4378.

3. Inanaga, J.; Hirata, K.; Saeki, H.; Katsuki, T.; Yamaguchi, M. A rapid esterification by mixed anhydride and its application to large-ring lactonization. Bull. Chem. Soc. Jpn. 1979, 52, 505-509.

4. Nicolaou, K.C.; Chakraborty, T.K.; Piscopio, A.D.; Minowa, N.; Bertinato, P. Total synthesis of rapamycin. J. Am. Chem. Soc. 1993, 115, 4419-4420.

5. Grubbs, R.H.; Miller, S.J.; Fu, G.C. Ring-closing metathesis and related processes in organic synthesis. Acc. Chem. Res. 1995, 28, 446-452. 
6. Deiters, A.; Martin, S.F. Synthesis of oxygen- and nitrogen-containing heterocycles by ringclosing metathesis. Chem. Rev. 2004, 104, 2299-2238.

7. Asaoka, M.; Abe, M.; Mukata, T.; Takei, H. Synthesis of macrocyclic lactones applying intramolecular 1,3-dipolar cycloaddition: synthesis of (+/-)-A26771B. Chem. Lett. 1982, 11, 215-218.

8. Kim, D.; Lee, J.; Shim, P.; Lim, J.I.; Doi, T.; Kim, S. Role of conformational effects on the regioselectivity of macrocyclic INOC reactions: Two new asymmetric total syntheses of $(+)-$ brefeldin A. J. Org. Chem. 2002, 67, 772-781.

9. Gothelf, K.V.; Jorgensen, K.A. Asymmetric 1,3-dipolar cycloaddition. Chem. Rev 1998, 98, 863-910.

10. Curran, D.P.; Fenk, C.J. Thermolysis of bis[2=[(trimethylsilyl)oxy]propyl]furoxan (TOP-furoxan). The first practical method for intermolecular cycloaddition of an in situ generated nitrile oxide with 1,2-di- and trisubstituted olefins. J. Am. Chem. Soc. 1985, 107, 6023-6028.

11. Sibi, M.P.; Itoh, K.; Jasperse, C.P. Chiral lewis acid catalysis in nitrile oxide cycloadditions. J. Am. Chem. Soc. 2004, 126, 718-719.

12. Shing, T.K.M.; Wong, W.F.; Cheng, H.M.; Kwok, W.S.; So, K.H. Intramolecular nitrile oxide-alkene cycloaddition of sugar derivatives with unmasked hydroxyl group(s). Org. Lett. 2007, 9, 753-756 and references cited therein.

13. Paek, S.-M.; Seo, S.-Y.; Kim, S.-H.; Jung, J.-W.; Lee, Y.-S.; Jung, J.-K.; Suh, Y.-G. Concise syntheses of (+)-macrosphelides A and B. Org. Lett. 2005, 7, 3159-3162.

14. Paek, S.-M.; Yun, H.; Kim, N.-J.; Jung, J.-W.; Chang, D.-J.; Lee, S.; Yoo, J.; Park, H.-J.; Suh, Y.-G. Concise syntheses of $(+)$-macrosphelides $\mathrm{A}$ and $\mathrm{B}$; studies on the macro-ring closure strategy. J. Org. Chem. 2009, 74, 554-561.

15. Yun, H.; Paek, S.-M.; Jung, J.-W.; Kim, N.-J.; Kim, S.-H.; Suh, Y.-G. First total syntheses of (-)macrosphelides $\mathrm{J}$ and $\mathrm{K}$ and elucidation of their absolute configuration Chem. Commun. 2009, 18, 2463-2465.

16. Sunazuka, T.; Hirose, T.; Harigaya, Y.; Takamatsu, S.; Hayashi, M.; Komiyama, K.; Ōmura, S.; Sprengeler, P.A.; Smith, A.B., III. Relative and absolute stereochemistries and total synthesis of (+)-macrosphelides A and B, potent, orally bioavailable inhibitors of cell-cell adhesion. J. Am. Chem. Soc. 1997, 119, 10247-10248.

17. Ono, M.; Nakamura, H.; Konno, F.; Akita, H. Total syntheses of macrosphelides (+)-A, (-)-A and (+)-E. Tetrahedron: Asymmetry 2000, 11, 2753-2764.

18. Kobayashi, Y.; Kumar, G.B.; Kurachi, T.; Acharya, H.P.; Yamazaki, T.; Kitazume, T. Furan ring oxidation strategy for the synthesis of macrosphelide A and B. J. Org. Chem. 2001, 66, 2011-2018.

19. Sharma, G.V.M.; Mouli, C.C. The total synthesis of macrosphelides A and E from carbohydrate precursors. Tetrahedron Lett. 2002, 43, 9159-9161.

20. Kusaka, S.-I.; Dohi, S.; Doi, T.; Takahashi, T. Total synthesis of macrosphelide A by way of palladium-catalyzed carbonylative esterification. Tetrahedron Lett. 2003, 44, 8857-8859.

21. Matsuya, Y.; Kawaguchi, T.; Nemoto, H. New strategy for the total synthesis of macrosphelides A and B based on ring-closing metathesis. Org. Lett. 2003, 5, 2939-2941. 
22. Takahashi, T.; Kusaka, S.-I.; Doi, T.; Sunazuka, T.; Omura, S. A combinatorial synthesis of a macrosphelide library utilizing a palladium-catalyzed carbonylation on a polymer support. Angew. Chem., Int. Ed. 2003, 42, 5230-5234.

23. Matsuya, Y.; Kawaguchi, T.; Ishihara, K.; Ahmed, K.; Zhao, Q.-L.; Kondo, T.; Nemoto, H. Synthesis of macrosphelides with a thiazole side chain: New antitumor candidates having apoptosis-inducing property. Org. Lett. 2006, 8, 4609-4612.

24. Matsuya, Y.; Nemoto, H. Artificial macrosphelides as a novel apoptosis-inducing compound. Heterocycles 2010, 81, 57-66 and references cited therein.

25. The regioisomer of the INOC product was not detected. This result matches with the previous observations reported in references [7] and [8].

26. Corey, E.J.; Xu, F.; Noe, M.C. A rational approach to catalytic enantioselective enolate alkylation using a structurally rigidified and defined chiral quaternary ammonium salt under phase transfer conditions. J. Am. Chem. Soc. 1997, 119, 12414-12415.

27. Park, H.-G.; Jeong, B.-S.; Yoo, M.-S.; Lee, J.-H.; Park, M.-K.; Lee, Y.-J.; Kim, M.-J.; Jew, S.-S. Highly enantioselective and practical cinchona-derived phase-transfer catalysts for the synthesis of $\alpha$-amino acids. Angew. Chem., Int. Ed. 2002, 41, 3036-3038.

28. A manuscript on detailed mechanism of the corresponding INOC and the synthetic application of isoxazoline 6 to the preparation of macrosphelide $\mathrm{J} \& \mathrm{~K}$ is in preparation.

Sample Availability: Samples of the compounds are available from the authors.

(C) 2011 by the authors; licensee MDPI, Basel, Switzerland. This article is an open access article distributed under the terms and conditions of the Creative Commons Attribution license (http://creativecommons.org/licenses/by/3.0/). 